\title{
Are congenital vertebral anomalies and spina bifida cystica aetiologically related?
}

\author{
R G LENDON,* RUTH WYNNE-DAVIES, $\dagger$ AND MEHROO LENDON $\ddagger$ \\ From the Departments of Anatomy* and Pathology ${ }_{+}^{+}$, University of Manchester, Manchester, and \\ $\dagger$ Department of Orthopaedic Surgery (Clinical Genetics Research), University of Edinburgh, Edinburgh
}

SUMMARY Radiological records of 104 patients with multiple vertebral anomalies without apparent spina bifida and 112 infants with spina bifida cystica were surveyed and the incidences of hemivertebrae and of rib, vertebral body, and vertebral arch fusions were recorded. The distributions of $\sigma_{\infty}^{\infty}$ these four anomalies along the vertebral axis were found not to be statistically different between the $\frac{i}{\mathrm{~N}}$

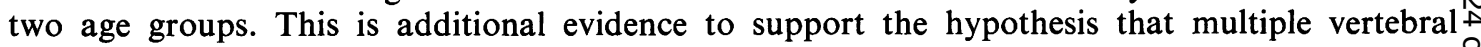
anomalies and anencephaly-spina bifida cystica are aetiologically related.

In a family survey of patients with congenital vertebral anomalies, Wynne-Davies ${ }^{1}$ found that the number of sibs affected with anencephaly or spina bifida cystica of index patients with multiple vertebral body anomalies (MVA) was much in excess of the general population frequency. Additional epidemiological evidence also showed similarities with previously published work relating to anencephalyspina bifida cystica (ASB). Wynne-Davies concluded that MVA without apparent spina bifida is aetiologically related to ASB, carrying a 5 to $10 \%$ risk to subsequent sibs of any one of these defects.

In a subsequent radiological study, Naik et $a l^{2}$ investigated the number and distribution of vertebral and rib anomalies in a group of children with spina bifida cystica and found characteristic distributions for the occurrence of hemivertebrae and rib and vertebral arch or body fusions. In view of these findings we thought it of interest to review the radiological records of patients with MVA without apparent spina bifida, whose genetic and epidemiological data had previously been studied by Wynne-Davies, and to compare these with the findings of Naik et al to see whether the distribution of anomalies was the same or different.

\section{Material and methods}

Radiological records were used from 104 of 118 patients previously classified as having MVA without spina bifida from scoliosis clinics in Edinburgh and London. ${ }^{1}$ The records rejected were from cases where Received for publication 1 December 1980 the deformity had been so severe that it had proved $\stackrel{\mathbb{}}{3}$

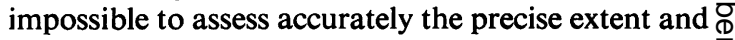
type of the malformations. The distributions of vertebral and rib anomalies in this group were $₫$ compared with post mortem radiological finding. from 112 infants with spina bifida cystica fro Sheffield and Manchester. ${ }^{2}$ All recorded radiologici defects from the thoracic and lumbar regions were noted, but as hemivertebrae, fused vertebral bodies and arches, and fused ribs were the commonest major $\mathbb{\perp}$ malformations in the two series this paper will $\vec{F}$ concentrate on these four categories of deformity.

In the occasional instances when a bilateral arch or $\bar{T}$ rib fusion was present at any level this was recorded as a single defect at that level rather than a double one, the assumption being made that a single developmental or subsequent pathological event was ${ }^{-}$ responsible for the bilaterality.

The results were expressed in two ways; firstly, as absolute numbers of malformations at each level and, $₹$ secondly, as a proportion at each level of the total 0 number of each type of malformation along the vertebral axis.

The distributions of the malformations were compared using the Kolmogonov-Smirnov test to see if they were significantly different.

\section{Results}

The total number of vertebral body fusions at each level in the two groups is shown in table 1 , vertebral arch fusions are given in table 2 , and rib fusions in table 3. A comparison of the proportion of these 
TABLE 1 Number of vertebral body fusions involving each vertebral level

\begin{tabular}{|c|c|c|c|c|c|c|c|c|c|c|c|c|c|c|c|c|c|c|}
\hline & $T I$ & 2 & 3 & 4 & 5 & 6 & 7 & 8 & 9 & 10 & 11 & 12 & (13) & $L I$ & 2 & 3 & 4 & 5 \\
\hline Scoliosis + MVA & 7 & 13 & 21 & 22 & 22 & 20 & 19 & 16 & 14 & 12 & 9 & 9 & (1) & 6 & 3 & 5 & 4 & 2 \\
\hline Spina bifida cystica & 2 & 1 & 3 & 5 & 4 & 4 & 4 & 5 & 3 & 2 & 3 & 2 & 一 & - & - & 1 & 1 & - \\
\hline
\end{tabular}

TABLE 2 Number of vertebral arch fusions involving each vertebral level

\begin{tabular}{|c|c|c|c|c|c|c|c|c|c|c|c|c|c|c|c|c|c|c|}
\hline & $T I$ & 2 & 3 & 4 & 5 & 6 & 7 & 8 & 9 & 10 & 11 & 12 & (13) & $L I$ & 2 & 3 & 4 & 5 \\
\hline Scoliosis + MVA & 4 & 5 & 7 & 11 & 14 & 15 & 20 & 19 & 17 & 11 & 4 & 6 & (1) & 7 & 4 & 4 & 2 & 1 \\
\hline Spina bifida cystica & 3 & 5 & 4 & 4 & 7 & 7 & 8 & 9 & 8 & 10 & 7 & 4 & - & 1 & $i$ & 1 & 4 & 3 \\
\hline
\end{tabular}

TABLE 3 Number of rib fusions involving each vertebral level

\begin{tabular}{lllllllllllllll}
\hline Rib & 1 & 2 & 3 & 4 & 5 & 6 & 7 & 8 & 9 & 10 & 11 & 12 & $(13)$
\end{tabular}

$\begin{array}{llllllllllllll}\text { Scoliosis + MVA } & 1 & 2 & 3 & 8 & 12 & 9 & 13 & 15 & 9 & 4 & 1 & 2 & \text { (1) }\end{array}$

$\begin{array}{llllllllllllll}\text { Spina bifida cystica } & 2 & 4 & 6 & 7 & 6 & 5 & 3 & 5 & 9 & 7 & 4 & 3 & -\end{array}$

three malformations occurring at each level is shown in figs 1 to 3 .

Equivalent figures for hemivertebrae are given in table 4. Table 4 is slightly different from the other tables because of the presence in the multiple vertebral anomalies group of occasional extra or supplementary hemivertebrae. ${ }^{3}$ These extra hemivertebrae have been designated T2A, T3A, etc, when present. This anomaly was not encountered in the spina bifida cystica group.

Using the Kolmogonov-Smirnov test the distributions of vertebral body, of vertebral arch, and of rib fusions were found not to be significantly different at the 0.05 probability level. Because of the presence of additional hemivertebrae this test was performed twice on the hemivertebra data, once comparing

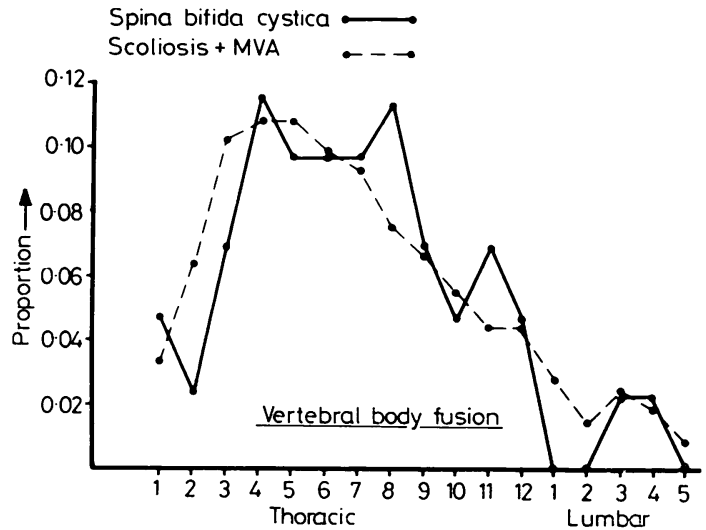

FIG 1 Proportion of vertebral body fusions at each vertebral level. numbers of hemivertebrae at each level ignoring the extra hemivertebrae, and once including the extra hemivertebrae in the calculations. The distributions were found not to be significantly different at the 0.05 probability level using either set of data.

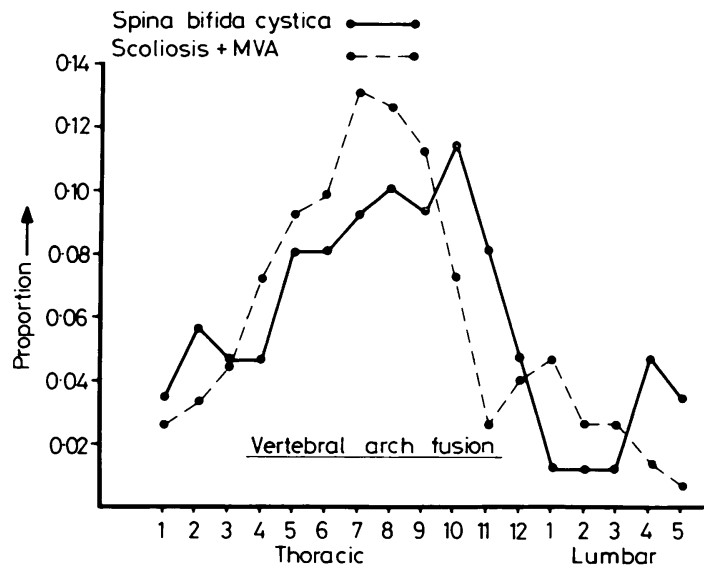

FIG 2 Proportion of vertebral arch fusions at each vertebral level.

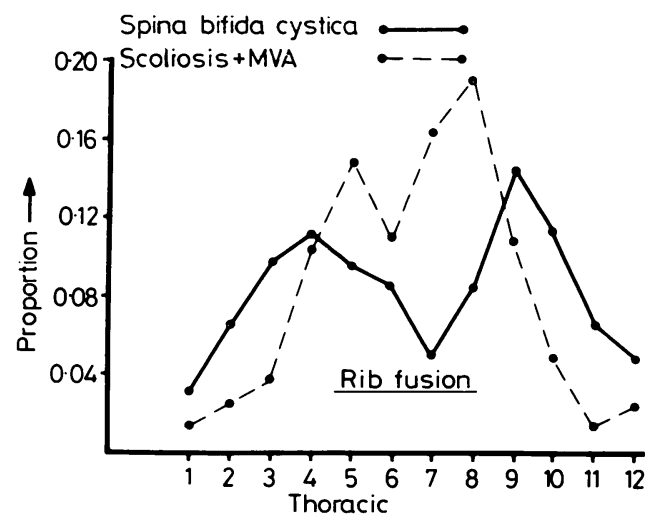

FIG 3 Proportion of rib fusions at each vertebral level. 


\section{Discussion}

The aim of this study was to see whether the dis- $\vec{F}$ tribution of vertebral and rib malformations in a group with spina bifida cystica was the same as or similar to the distribution of such anomalies in a group with multiple vertebral defects. If the distributions were similar it would be additional evidence to support Wynne-Davies's suggestion ${ }^{1} \infty$ that multiple vertebral anomalies and spina bifida- $\overrightarrow{0}$ anencephaly are aetiologically related. The results appear to support such a relationship. The dis- $\vec{\omega}$ tribution of vertebral body fusions was very similar in the two groups (fig 1) and so too was the distribu- 0 tion of vertebral arch fusions (fig 2). In both cases $\vec{\infty}$ the statistical test employed did not disprove the hypothesis.

Although the distribution of rib fusions looked superficially different (fig 3 ), the two series were not 음 found to be statistically significantly different. In two previous studies on rib malformations in spina bifida cystica cases, ${ }^{24}$ a peak for rib malformations $\overparen{\varnothing}$ was found at $\mathrm{T} 4$, and in the latter study a second $\bar{Z}$

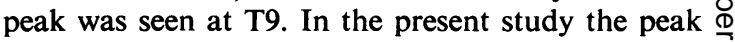
incidences for rib fusions in cases with MVA were $\vec{\bullet}$ at T5 and T8. One possibility for the closer proximit $\infty$ of these peaks in the MVA groups could be th presence of the scoliosis, as this would tend to pusid. ribs together on the inside of the curve and thus leat to secondary fusions.

The distribution of hemivertebrae was confused by

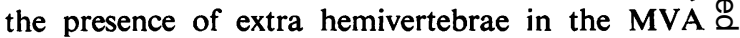
group. It can be seen (table 4) that in both series the $\overrightarrow{\overrightarrow{0}}$ defects tend to be grouped in the upper and lower 3 thoracic regions. However, there is an additional peak around T6 in the MVA group. The two distributions were not significantly different, however, using the Kolmogonov-Smirnov test.

Although there have been surveys of the incidences $\frac{5}{3}$ of vertebral and rib anomalies in adults and children, ${ }^{56}$ it is rare for details of the incidences of $O$ particular malformations at individual vertebral levels to be recorded in a large series of cases. This 음 makes it difficult to decide whether the results we $>$ have obtained, which suggest that the patterns of 을. vertebral and rib anomalies in our two groups were $N$ similar, represents an aetiological link, or whether o the same distribution patterns occur in the normal 0 population, but at lower frequencies. $\operatorname{Etter}^{5} \mathrm{~N}$ fortunately recorded very precisely the incidences of $\bar{Z}$ different types of rib anomalies at each level. Hiso peak incidence for rib fusions was between ribs 1 and $\overparen{\varnothing}$ 2 , and his overall distribution pattern was very $\stackrel{\oplus}{+}$ different from our observations. This suggests that, $\underset{T}{T}$ for one type of anomaly at least, our findings do not 
just represent an increased frequency of a normal spontaneously occurring malformation.

We conclude that the distributions of the four types of vertebral and rib malformations we have studied were not significantly different between the two groups. Consequently, we suggest that this does not disprove the hypothesis that multiple vertebral anomalies and anencephaly-spina bifida cystica are aetiologically related.

\section{References}

1 Wynne-Davies R. Congenital vertebral anomalies: aetiology and relationship to spina bifida cystica. $J$ Med Genet $1975 ; 12: 280-8$.
2 Naik DR, Lendon RG, Barson AJ. A radiological study of vertebral and rib malformations in children with myelomeningocele. Clin Radiol 1978;29:427-30.

3 Shanks SC, Kerley P. A textbook of X-ray diagnosis. 4th ed. London: Lewis, 1971.

4 McLennan JE. Rib anomalies in myelodysplasia. Biol Neonate 1976;29:129-49.

5 Etter LE. Osseous abnormalities of the thoracic cage seen in forty thousand consecutive chest photoroentgenograms. AJR 1944;51:359-63.

6 Shands AR, Bundens WD. Congenital deformities of the spine. An analysis of the roentgenograms of 700 children. Bull Hosp Joint Dis 1956;17:110-33.

Requests for reprints to Dr R G Lendon, Department of Anatomy, University of Manchester, Stopford Building, Oxford Road, Manchester M13 9PT. 